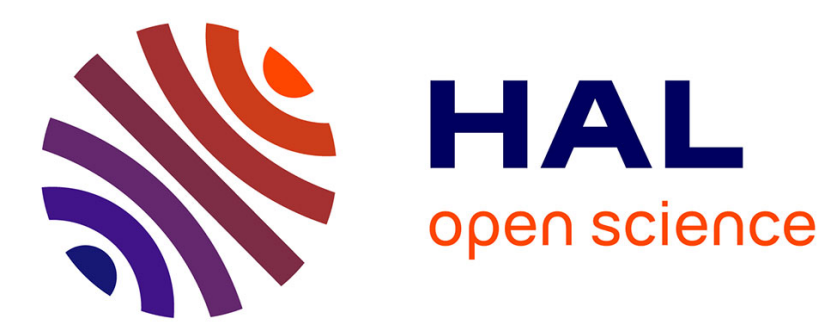

\title{
Some specific features and consequences of the thermal response of rubber under cyclic mechanical loading
}

Xavier Balandraud, Jean-Benoit Le Cam

\section{To cite this version:}

Xavier Balandraud, Jean-Benoit Le Cam. Some specific features and consequences of the thermal response of rubber under cyclic mechanical loading. Archive of Applied Mechanics, 2014, 84 (6), pp.773-788. 10.1007/s00419-014-0832-3 . hal-01005745

\section{HAL Id: hal-01005745 \\ https://hal.science/hal-01005745}

Submitted on 13 Jun 2014

HAL is a multi-disciplinary open access archive for the deposit and dissemination of scientific research documents, whether they are published or not. The documents may come from teaching and research institutions in France or abroad, or from public or private research centers.
L'archive ouverte pluridisciplinaire HAL, est destinée au dépôt et à la diffusion de documents scientifiques de niveau recherche, publiés ou non, émanant des établissements d'enseignement et de recherche français ou étrangers, des laboratoires publics ou privés. 


\title{
Some specific features and consequences of the thermal response of rubber under cyclic mechanical loading
}

the date of receipt and acceptance should be inserted later

\begin{abstract}
The present paper deals with the specificities of the thermal response of rubber under cyclic mechanical loading at constant ambient temperature. This question is important, since the stabilized thermal response is used in fatigue life criteria, especially for the fast evaluation of fatigue life. For this purpose, entropic coupling in a thermo-hyperelastic framework is first used to predict the variation in the heat source produced or absorbed by the material during cyclic loading. The heat diffusion equation is then used to deduce temperature variations under adiabatic and non-adiabatic conditions. The influence of several parameters on the stabilized thermal response is studied: signal shape, frequency, minimum and maximum stretch levels, multiaxiality of the mechanical state. The results show that, in the steady-state regime, the mean value between the maximum and minimum temperature variations over a mechanical cycle is different from zero. This is due to the specific variation in the heat source, which depends on both the stretch rate and the stretch level. This result has numerous consequences, in particular for fatigue. Indeed, the stabilized mean value between the maximum and minimum temperature variations during fatigue tests does not reflect only fatigue damage, since the entropic coupling also leads to a value different from zero. This is a major difference with respect to materials exhibiting only isentropic coupling, such as metallic materials.
\end{abstract}

Keywords Rubber elasticity · thermomechanical couplings · heat source $\cdot$ heat diffusion equation.

\section{Introduction}

Elastomers are polymeric materials that can undergo large deformations without breaking, owing to the ability of their constituent polymeric chains to rotate about the chain bonds. They are used in many fields of application, such as mechanical engineering, automotive engineering and aerospace engineering, due to their high elasticity, high damping and high elongation at failure. However, many phenomena involved in the deformation process of such materials are still not fully understood. We can cite, for instance, phenomena such as: stress softening, referred to as the Mullins effect $[1 ; 2 ; 3]$ in the

X. Balandraud

CLERMONT UNIVERSITÉ, Institut Français de Mécanique Avancée, Institut Pascal, BP 10448, 63000 Clermont-Ferrand, France

CNRS, UMR 6602, Université Blaise Pascal, Institut Pascal, 63171 Aubière, France

Tel.: +33-47-3288089

Fax: +33-47-3288100

E-mail: xavier.balandraud@ifma.fr

J.-B. Le Cam

UNIVERSITÉ DE RENNES 1, Institut de Physique de Rennes, UMR 6251 - CNRS/Université de Rennes 1, Campus de Beaulieu, 35042 Rennes, France

Tel.: +33-2-3235741

Fax: +33-2-3236100

E-mail: jean-benoit.lecam@univ-rennes1.fr 
literature, the Payne (or Fletcher-Gent) effect $[4 ; 5 ; 6 ; 7 ; 8]$, cavitation $[9 ; 10 ; 11]$ and stress-induced crystallization $[12 ; 13 ; 14 ; 15 ; 16]$. Classically, rubber-like elasticity is described as an entropic effect [17], which leads to heat production (absorption) during loading (unloading). The first experimental observations of this effect in the literature were those obtained by Gough ${ }^{1}$ [18] and Joule [19], and dealt with the increase in temperature of rubber during stretching. In spite of these first results, no further experimental study was fully dedicated to the thermomechanical characterization of elastomers, although this is a key point to study and to predict phenomena such as fatigue damage $[20 ; 21 ; 22$; 23], and to establish material constitutive equations. From a theoretical point of view, the work by Chadwich and Creasy [24] is one of the very few studies to echo the experimental study of Joule. Until now, no study has allowed us to link this theoretical modeling with experimental results, even for homogeneous thermomechanical fields. It can be noted that new characterization methods in this field have recently been developed and applied to rubbers by coupling optical and infrared thermography measurements. These methods provide full kinematic and thermal fields without any contact with the rubber specimen under study $[10 ; 11 ; 25 ; 26 ; 27 ; 28 ; 29 ; 31 ; 32]$ and their coupling allows us to calculate heterogeneous heat source fields (typically at the crack tip [25]). In the future, the comparison between such experimental fields and adequate theoretical approaches should be of wide interest to the understanding of the thermomechanics of rubbers.

The present study deals with the predicted temperature variation in rubber under cyclic loading at constant ambient temperature. The heat sources produced or absorbed by the stretched material are obtained from the loading conditions by using the framework of continuum thermodynamics [33; 34] with isotropic thermo-hyperelastic behaviour. The heat sources considered here are only due to entropic coupling. The temperature variations are then deduced from the heat sources by solving the heat diffusion equation. For the sake of processing simplicity (in order to avoid full 3D calculations), applications are performed here with homogeneous deformation fields. This approach does not alter the conclusions of the study and enables us to focus on the specificities of the thermal response of rubber.

The paper is composed of four sections. The general thermomechanical framework and the heat diffusion equation are presented in Section 2. Section 3 provides some hypotheses to predict temperature variation during the deformation process. The numerical simulation setup is presented in Section 4, including the loading conditions of the cyclic tests to be simulated. Section 5 is devoted to the analysis of the results, for adiabatic and non-adiabatic conditions. Finally, before summarizing the results obtained, some remarks are made concerning the effect of the volumetric part of the heat source (effect of internal energy variation) on the thermal response.

\section{Thermomechanical framework}

\subsection{Thermodynamics with internal variables}

It is assumed that any thermomechanical system out of equilibrium can be considered as the sum of several homogeneous subsystems at equilibrium. In this context, constitutive equations are established by considering the deformation process as issuing from a quasi-static thermodynamic process. This process can be dissipative. The equilibrium state of each volume material element is defined by $n$ state variables: the absolute temperature $T$, the deformation gradient tensor $\mathbf{F}$ and $m(=n-2)$ internal tensorial variables $\boldsymbol{\xi}_{\alpha}$. When the free-energy function $\Psi$ is chosen as the Helmholtz free-energy function, it is written in terms of internal energy $e\left(\mathbf{F}, \eta, \boldsymbol{\xi}_{\boldsymbol{\alpha}}\right)$ and entropy $\eta\left(\mathbf{F}, T, \boldsymbol{\xi}_{\boldsymbol{\alpha}}\right)$ as follows:

$$
\Psi\left(\mathbf{F}, T, \boldsymbol{\xi}_{\boldsymbol{\alpha}}\right)=e\left(\mathbf{F}, \eta, \boldsymbol{\xi}_{\boldsymbol{\alpha}}\right)-T \eta\left(\mathbf{F}, T, \boldsymbol{\xi}_{\boldsymbol{\alpha}}\right)
$$

The three quantities $\Psi, e$ and $\eta$ refer to a unit volume of the reference configuration.

The local version of the second principle of thermodynamics leads to the local form of the ClausiusDuhem inequality in the reference configuration:

$$
\mathbf{P}: \dot{\mathbf{F}}-\rho(\dot{e}-T \dot{\eta})-\frac{1}{T} \mathbf{Q} \cdot \operatorname{Grad} T \geqslant 0
$$

\footnotetext{
1 "If one end of a slip of Caoutchouc be fastened to a rod of metal or wood, and a weight be fixed to the other extremity, in order to keep it in a vertical position; the thong will be found to become shorter with heat and longer with cold", Gough, 1805
} 
where $\mathbf{P}$ is the first Piola-Kirchhoff stress tensor (or nominal stress tensor) and $\mathbf{Q}$ the Piola-Kirchhoff heat flux (or nominal heat flux). $\rho$ is the density of the material in the reference configuration. Here, a point placed above a symbol denotes the temporal derivative of the considered quantity. The operators "." and ":" are the scalar product (dot product) and the tensorial product (trace of the matrix product), respectively. Note that the external heat source due to radiation, commonly denoted $R$, has been removed from the local form of the Clausius-Duhem inequality by using the local form of the balance of energy.

The term $-\frac{1}{T} \mathbf{Q} \cdot \operatorname{Grad} T$ (related to heat conduction) corresponds to the thermal dissipation $\mathcal{D}_{\text {the }}$. Based on physical observations, heat flows from the warmer to the colder region of a body. It is assumed that heat conduction follows Fourier's law :

$$
\mathbf{Q}=-\kappa_{0} \operatorname{Grad} T,
$$

where $\boldsymbol{\kappa}_{\mathbf{0}}$ is a positive semi-definite tensor characterizing the thermal conductivity of the material. As the absolute temperature $T$ is considered as non-negative, it follows that:

$$
\mathcal{D}_{\text {the }}=-\frac{1}{T} \mathbf{Q} \cdot \operatorname{Grad} T \geqslant 0
$$

The quantity $\mathbf{P}: \dot{\mathbf{F}}-\rho(\dot{e}-T \dot{\eta})$ corresponds to the intrinsic dissipation $\mathcal{D}_{\text {int }}$ (also named mechanical dissipation). It is considered as non-negative. Thus,

$$
\mathcal{D}_{i n t}=\mathbf{P}: \dot{\mathbf{F}}-\rho(\dot{e}-T \dot{\eta}) \geqslant 0
$$

This quantity is related to any irreversible mechanical mechanism (for instance fatigue damage, internal friction, viscosity...). Note that any change in the material microstructure may also have a part of mechanical irreversibility. The quantity is also written [35]:

$$
\mathcal{D}_{i n t}=\mathbf{P}: \dot{\mathbf{F}}-\rho\left(\frac{\partial \Psi}{\partial \mathbf{F}}\right)_{T, \boldsymbol{\xi}_{\alpha}}: \dot{\mathbf{F}}-\rho\left(\frac{\partial \Psi}{\partial T}\right)_{\mathbf{F}, \boldsymbol{\xi}_{\alpha}} \dot{T}-\rho \sum_{\beta=1}^{m}\left(\frac{\partial \Psi}{\partial \boldsymbol{\xi}_{\boldsymbol{\beta}}}\right)_{\mathbf{F}, T, \xi_{\alpha} \neq \xi_{\beta}}: \dot{\boldsymbol{\xi}_{\boldsymbol{\beta}}} \geqslant 0
$$

In the present formulation, constitutive state equations derive from the Helmholtz free energy function:

$$
\begin{aligned}
\eta & =-\left(\frac{\partial \Psi}{\partial T}\right)_{\mathbf{F}, \boldsymbol{\xi}_{\alpha}} \\
\mathbf{P}^{r e v} & =\rho\left(\frac{\partial \Psi}{\partial \mathbf{F}}\right)_{T, \boldsymbol{\xi}_{\boldsymbol{\alpha}}} \\
\boldsymbol{A}_{\boldsymbol{\beta}} & =\rho\left(\frac{\partial \Psi}{\partial \boldsymbol{\xi}_{\boldsymbol{\beta}}}\right)_{\mathbf{F}, T, \xi_{\alpha} \neq \xi_{\beta}}
\end{aligned}
$$

where $\mathbf{P}^{r e v}$ and $\boldsymbol{A}_{\boldsymbol{\beta}}$ are the thermodynamic forces associated with variables $\mathbf{F}$ and $\boldsymbol{\xi}_{\boldsymbol{\beta}}$, respectively. Note that in the case of a reversible transformation (irreversible part $\mathbf{P}^{\text {irrev }}$ of the stress equal to zero), we have:

$$
\mathbf{P}=\mathbf{P}^{r e v}=\rho\left(\frac{\partial \Psi}{\partial \mathbf{F}}\right)_{T, \boldsymbol{\xi}_{\alpha}}
$$

\subsection{Calorimetry}

The specific heat capacity, denoted $C_{\mathbf{F}, \boldsymbol{\xi}_{\alpha}}$ in the following, is the energy required to produce a unit increase in temperature of a unit reference volume of a body while maintaining the deformation and internal variables fixed. It is defined by:

$$
C_{\mathbf{F}, \boldsymbol{\xi}_{\alpha}}=-T\left(\frac{\partial^{2} \Psi}{\partial T \partial T}\right)_{\mathbf{F}, \boldsymbol{\xi}_{\alpha}}>0
$$

In the following, the specific heat capacity is considered as constant during the deformation process and is simply denoted $C$. 
2.3 Heat diffusion equation

Using the two principles of thermodynamics, the local form of the balance of energy and convenient entropy form are respectively written in the material description:

$$
\begin{aligned}
\rho \dot{e} & =\mathbf{P}: \dot{\mathbf{F}}-\operatorname{Div} \mathbf{Q}+\mathbf{R} \\
\rho T \dot{\eta} & =-\operatorname{Div} \mathbf{Q}+\mathcal{D}_{\text {int }}+\mathbf{R}
\end{aligned}
$$

where $R$ is the external heat source (radiation, for instance).

From Eq. 7, we obtain:

$$
\rho T \dot{\eta}=-\rho T\left(\frac{\partial \dot{\Psi}}{\partial T}\right)=-\rho T \frac{\partial^{2} \Psi}{\partial T \partial T} \dot{T}-\rho T \frac{\partial^{2} \Psi}{\partial T \partial \mathbf{F}}: \dot{\mathbf{F}}-\rho T \sum_{\beta=1}^{m} \frac{\partial^{2} \Psi}{\partial T \partial \boldsymbol{\xi}_{\boldsymbol{\beta}}}: \dot{\boldsymbol{\xi}_{\boldsymbol{\beta}}}
$$

As mentioned previously, the term $-T \frac{\partial^{2} \Psi}{\partial T \partial T}$ corresponds to the specific heat capacity $C$.

According to Eqs. 8, 9 and 10, we have: $\rho \frac{\partial^{2} \Psi}{\partial T \partial \mathbf{F}}=\frac{\partial \mathbf{P}}{\partial T}, \rho \frac{\partial^{2} \Psi}{\partial T \partial \boldsymbol{\xi}_{\boldsymbol{\beta}}}=\frac{\partial \boldsymbol{A}_{\boldsymbol{\beta}}}{\partial T}$, and consequently Eq. 14 can be re-written:

$$
\rho T \dot{\eta}=\rho C \dot{T}-T \frac{\partial \mathbf{P}}{\partial T}: \dot{\mathbf{F}}-T \sum_{\beta=1}^{m} \frac{\partial \boldsymbol{A}_{\boldsymbol{\beta}}}{\partial T}: \dot{\boldsymbol{\xi}_{\boldsymbol{\beta}}}
$$

The local heat diffusion equation derives from Eqs. 12, 13 and 15. It is written using Eq. 3:

$$
\rho C \dot{T}-\operatorname{Div}\left(\boldsymbol{\kappa}_{\mathbf{0}} \operatorname{Grad} T\right)=\mathcal{D}_{i n t}+T \frac{\partial \mathbf{P}}{\partial T}: \dot{\mathbf{F}}+T \sum_{\beta=1}^{m} \frac{\partial \boldsymbol{A}_{\boldsymbol{\beta}}}{\partial T}: \dot{\boldsymbol{\xi}_{\boldsymbol{\beta}}}+R
$$

Let us denote $s_{h}$ the overall heat source ${ }^{2}$ induced by the deformation process:

$$
s_{h}=\mathcal{D}_{i n t}+T \frac{\partial \mathbf{P}}{\partial T}: \dot{\mathbf{F}}+T \sum_{\beta=1}^{m} \frac{\partial \boldsymbol{A}_{\boldsymbol{\beta}}}{\partial T}: \dot{\boldsymbol{\xi}_{\boldsymbol{\beta}}}
$$

The terms $T \frac{\partial \mathbf{P}}{\partial T}: \dot{\mathbf{F}}$ correspond to the heat source due to entropic coupling. It is denoted $s_{e c}$ in the following. The term $T \frac{\partial \boldsymbol{A}_{\boldsymbol{\beta}}}{\partial T}: \dot{\boldsymbol{\xi}_{\boldsymbol{\beta}}}$ corresponds to the other thermomechanical couplings (for instance related to phase change in the material) and is denoted $s_{\text {otc }}$ in the following. Thus the overall heat source is re-written:

$$
s_{h}=\mathcal{D}_{\text {int }}+s_{\text {ec }}+s_{\text {otc }}
$$

Finally, the heat diffusion equation is written:

$$
\rho C \dot{T}-\operatorname{Div}\left(\kappa_{0} \operatorname{Grad} T\right)=s_{h}+R
$$

\section{Prediction of temperature variation during the deformation process}

Section 3.1 gives some assumptions required to simplify the heat diffusion equation in the case of homogeneous deformation fields. These assumptions seek to simplify the calculations and do not alter the conclusions of the study. Section 3.2 presents the thermo-hyperelasticity framework which enables us to define the heat sources $s_{e c}$ due to entropic coupling from the loading conditions.

\footnotetext{
${ }^{2}$ The term 'heat source' is used in this paper to mean the heat power density which is produced or absorbed by the material. Note that the terms 'heat source' and 'heat' must be distinguished. The heat (in $\mathrm{J} / \mathrm{m}^{3}$ ) is the temporal integration of the heat source $\left(\right.$ in $\mathrm{W} / \mathrm{m}^{3}$ ).
} 


\subsection{Simplified heat diffusion equation}

Let us consider an instance of mechanical loading such that the deformation field is homogeneous in the specimen at any time. Due to the very small thermal diffusivity of elastomers (order of magnitude of $\left.10^{-7} \mathrm{~m}^{2} \cdot \mathrm{s}^{-1}\right)$, the temperature field is almost homogeneous in the specimen at any time. In fact, temperature gradients exist close to the specimen boundary. These are related to the heat exchanges with the surroundings. However, these small temperature gradients do not prevent the temperature at the surface from being nearly equal to the mean temperature in the specimen. Let us denote $\theta$ the temperature variation with respect to the equilibrium temperature $T^{r e f}$ in the reference state, corresponding to the undeformed state ( $T^{r e f}$ is equal to the ambient temperature). The heat exchanges at the specimen boundary are linearized assuming that the temperature variations $\theta$ are small. After some calculations which are not detailed here, the 3D heat diffusion equation can be rewritten as follows $[36 ; 37 ; 38 ; 39 ; 40 ; 41 ; 31 ; 32]$ :

$$
\rho C\left(\dot{\theta}+\frac{\theta}{\tau}\right)=s_{h}
$$

where $\tau$ is a time constant characterizing the heat exchanges between the specimen and its surroundings. This formulation can be referred to as the "0D" formulation. For similar simplifications of the heat diffusion equation, the reader can also refer to Refs. [42; 43;44;45;46;47], which are devoted to wires or thin plates, leading respectively to $1 \mathrm{D}$ or $2 \mathrm{D}$ formulations.

It is worth noting that, experimentally, $\tau$ depends on the specimen material (through its thermal diffusivity), the environment (ambient air and material used for the grips of the testing machine) and the specimen geometry (through the surface-area-to-volume ratio). For instance, the value of $\tau$ depends on the air flow around the specimen: the higher the convection, the smaller the parameter $\tau$. Let us note that changes in the specimen shape (due to large deformations) modify the surface-area-to-volume ratio. The area of the surface in contact with the grips of the testing machine and the material of the grips also have an influence. A value of $\tau$ should be measured for each testing configuration (machine used, environment, stretch level, etc). Experimentally, $\tau$ is usually identified using a natural return to room temperature. Indeed, in this case the temperature variation is governed by the heat diffusion equation, with no heat source produced by the material itself $(\dot{\theta}+\theta / \tau=0)$. In the present numerical study, $\tau$ is considered as a constant. For the experimental characterization of $\tau$, the reader can refer to Ref. [31].

The value of $\tau$ characterizes the level of non-adiabaticity of the mechanical test. If $\tau=0$, the test is isothermal (the heat produced or absorbed by the material due to stretching is instantaneously exchanged with the outside). A perfectly adiabiatic evolution corresponds to $\tau=\infty$. More generally, if $\tau$ is much larger than the test duration, the evolution can be considered as adiabatic over this duration.

\subsection{Thermally and mechanically isotropic hyperelasticity}

In the present study, no intrinsic dissipation is taken into account, meaning that $\mathcal{D}_{\text {int }}=0$. Only the contribution of entropic coupling is considered in the heat source expression, i.e. $s_{h}=s_{e c}$. Such material behaviour is observed for unfilled rubbers, for instance in silicone rubbers that exhibit a perfect non-linear and reversible behaviour with neither Mullins effect nor hysteresis loop, meaning that all loading and unloading curves are superimposed [50].

The prediction of the heat sources produced during the deformation process requires the choice of a free energy density $\Psi$. Here, the material is assumed to behave as a hyperelastic material that is mechanically incompressible. As high strain levels are not investigated here, the Neo-Hookean model is chosen [51]. The free energy function is then given by the following strain energy density:

$$
\rho \Psi(\mathbf{F}, T)=W(\mathbf{F}, T)=\frac{1}{2} N k T\left(I_{1}-3\right)
$$

where $N$ is the number of network chains per unit volume, $k$ is Boltzmann's constant, $T$ is the temperature in Kelvin and $I_{1}$ is the first invariant of the left Cauchy-Green deformation tensor $\mathbf{F F}^{T}$. 
If the material is mechanically incompressible, the deformation gradient tensor for a biaxial loading is given by:

$$
F=\lambda e_{1} \otimes e_{1}+\lambda^{B} e_{2} \otimes e_{2}+\lambda^{-(B+1)} e_{3} \otimes e_{3}
$$

where:

- $e_{1}, e_{2}$ and $e_{3}$ are three orthonormal vectors of the 3D euclidian space,

$-\lambda$ is the stretch ratio in the $e_{1}$ direction, defined as the ratio of the current length to the initial length in the $e_{1}$ direction,

- the operator $\otimes$ between two vectors is such that $[a \otimes b]_{i j}=a_{i} b_{j}$,

$-B$ is the coefficient of biaxiality [48; 49]. It is equal to $-0.5,0$ and 1 for uniaxial tension, pure shear and equibiaxial tension, respectively.

The strain energy density is written:

$$
W(\lambda, T)=\frac{1}{2} N k T\left[\lambda^{2}+\lambda^{2 B}+\lambda^{-2(B+1)}-3\right]
$$

The heat source is then given by:

$$
s_{h}=s_{e c}=T \frac{\partial \mathbf{P}}{\partial T}: \dot{\mathbf{F}}=T \frac{\partial^{2} W}{\partial T \partial \lambda} \frac{d \lambda}{d t}
$$

Using this expression, the heat diffusion equation is:

$$
\rho C\left(\dot{\theta}+\frac{\theta}{\tau}\right)=N k T\left[\lambda+B \lambda^{2 B-1}-(B+1) \lambda^{-2 B-3}\right] \frac{d \lambda}{d t}
$$

Remark 1 - The quantity $N k T$ appearing in the heat source expression contains the value of the temperature in Kelvin. For temperature variations which do not exceed a few degrees, $N k T$ remains in fact nearly equal to $N k T^{r e f}$, where $T^{r e f}$ is the initial temperature (in other words, the temperature variations have no significant influence on the elastic properties of the material). For instance, a temperature variation of 1 degree around ambient temperature leads to a heat source variation of $100 \times 1 / 293=0.34 \%$.

For uniaxial tension, pure shear and equibiaxial tension, we have respectively:

$$
\begin{aligned}
& \rho C\left(\dot{\theta}+\frac{\theta}{\tau}\right)=N k T^{\text {ref }}\left(\lambda-\frac{1}{\lambda^{2}}\right) \frac{d \lambda}{d t} ; \\
& \rho C\left(\dot{\theta}+\frac{\theta}{\tau}\right)=N k T^{r e f}\left(\lambda-\frac{1}{\lambda^{3}}\right) \frac{d \lambda}{d t} ;
\end{aligned}
$$

and

$$
\rho C\left(\dot{\theta}+\frac{\theta}{\tau}\right)=2 N k T^{r e f}\left(\lambda-\frac{1}{\lambda^{5}}\right) \frac{d \lambda}{d t}
$$

Remark 2 - The heat source due to entropic coupling depends on the stretch rate $d \lambda / d t$, but also on the stretch ratio through the term $\lambda+B \lambda^{2 B-1}-(B+1) \lambda^{-2 B-3}$. This is a major difference compared to materials exhibiting only an isentropic-type thermoelastic coupling (metallic materials in particular). In this latter case, the heat source depends only on the strain rate. This property has inspired the so-called thermoelastic stress analysis technique [52], which enables stress distribution to be measured in metallic structural components from full temperature field measurements. The equations written in

this section are used below to predict the thermal response of rubber for different loading conditions. Section 4 presents the numerical setup, including in particular the different tests to be simulated. 


\section{Numerical simulation setup}

This section describes the different tests and provides some information about the numerical solution of the heat diffusion equation.

Material parameter - The material is assumed to behave as a neo-Hookean material. In this case, the only material parameter is the number of chain segments $N$ per unit volume. As the temperature variations in the simulations below will not exceed a few degrees, the quantity $N k T$ is considered as constant (see Remark 1 above). It is taken as equal to $2 \mathrm{MPa}$. This is the order of magnitude obtained from experimental tests. This choice has been already done in previous theoretical studies (see for instance Ref. [30]).

Level of non-adiabaticity of the thermodynamic evolution - Two series of tests are simulated below: one under adiabatic condition and another under non-adiabatic conditions. The adiabatic case corresponds to a time constant $\tau$ which is infinite. In practice, the term $\theta / \tau$ is removed from the heat diffusion equation. For the non-adiabatic case, the time constant $\tau$ is taken as equal to $60 \mathrm{~s}$ in the present study. This is the order of magnitude of $\tau$ measured for a $1 \times 5 \times 20 \mathrm{~mm}^{3}$ natural rubber specimen (see for instance Refs. [31] and [32]).

Loading conditions - Cyclic tests are considered here. Several parameters are studied: shape of the signal applied, frequency, strain ratio and multiaxiality of the mechanical state. Two types of signal are considered: triangular and sinusoidal. The former case enables us to exclude the influence of the stretch rate variation from the analysis $(d \lambda / d t$ is constant during the loading stage and the unloading stage). The latter case is classically used for experimental cyclic tests.

Tables 1 and 2 summarize the different uniaxial tests to be carried out, for adiabatic and nonadiabatic conditions respectively. The first two columns give the minimum and maximum stretch ratios applied, $\lambda_{\min }$ and $\lambda_{\max }$ respectively. The strain ratio $R_{\epsilon}$ is defined as the ratio between the minimum strain and the maximum strain. $f$ is the frequency of the cyclic mechanical loading. The main objective of the present study is to investigate the stabilized thermal response, which is obtained after a certain period of time. For adiabatic conditions, the stabilization of the temperature oscillation is obtained from the first mechanical cycle. For non-adiabatic conditions, the stabilized oscillation of the temperature is reached after a duration which can be approximated to $3 \tau$.

- Tests \#1 and \#2 correspond to uniaxial tension (UT) loading, processed under adiabatic conditions. These tests aim at highlighting the effect of the signal shape (triangular or sinusoidal) on the heat source and temperature variations under adiabatic conditions, for which Eq. 20 becomes $\rho C \dot{\theta}=s_{h}$. As a consequence, the loading frequency does not influence to the temperature variation level (It is arbitrarily fixed to $0.5 \mathrm{~Hz}$ for both simulations).

- Tests \#3 to \#8 enable us to study the effect of the loading parameters under non-adiabatic conditions. Apart from test \#5, the loading frequency is equal to $0.5 \mathrm{~Hz}$ and the test duration is chosen equal to $300 \mathrm{~s}$, which corresponds to $5 \tau$. This enables us to observe both the transient and stabilized regimes. Test \#5 is a special case, considered to study the effect of the loading frequency. Several frequencies in the range $[0.001 \mathrm{~Hz} ; 0.2 \mathrm{~Hz}]$ are tested. This range enables us to study the influence of the heat exchanges with the outside during one mechanical cycle. Indeed, the longer the period $1 / f$ of the mechanical cycle, the stronger the effect of the heat exchanges with the outside environment during the cycle. The test duration is adapted to the tested frequency in order to observe several cycles. In the table, UT, EQT and PS correspond to uniaxial tension, equibiaxial tension and pure shear, respectively.

Data to be extracted from the temperature variations - Under non-adiabatic conditions, the thermal response evolves from one cycle to another before stabilizing. In order to follow the global thermal response, we define by $\hat{\theta}$ the mean value between the maximum and minimum temperature variations over a mechanical cycle by:

$$
\hat{\theta}=\frac{\theta_{\max }+\theta_{\min }}{2}
$$

where $\theta_{\min }$ and $\theta_{\max }$ are the minimum and maximum temperature variations over a mechanical cycle. Moreover, the mean temperature variation over one cycle, defined as the temporal integration of $\theta$ over the cycle, is also calculated for each cycle. 
Numerical resolution - The resolution of Eq. 26, Eq. 27 and Eq. 28 is based on an implicit Euler scheme. Particular attention is paid to time discretization, since accurate results require a very refined temporal discretization. The number of time steps per mechanical cycle is critical. After some preliminary tests, this number is fixed to $10^{4}$. Such a refined temporal discretization is particularly required for triangular loading, which exhibits a non-continuous variation in the heat source, induced by the non-continuous variation of $d \lambda / d t$.

Unit used for heat sources - The heat source $s_{h}$ is expressed in W.m ${ }^{-3}$. However, it can be useful to divide this quantity by $\rho C$ :

$$
s=\frac{s_{h}}{\rho C}
$$

In the following parts of the paper, the term "heat source" is still used for the quantity $s$. This later is expressed in ${ }^{\circ} \mathrm{C} . \mathrm{s}^{-1}$. Using this unit enables us to directly read the temperature rate in the case of adiabatic evolution. Obviously, in case of non-adiabatic conditions, the temperature rate is different from the heat source $s$ because of the heat exchanges with the outside.

From Eq. 20, the heat diffusion equation becomes:

$$
\dot{\theta}+\frac{\theta}{\tau}=s
$$

The heat is the temporal integration of the heat sources. When divided by $\rho C$, the heat is expressed in ${ }^{\circ} \mathrm{C}$.

\section{Results and analysis}

\subsection{Tests under adiabatic conditions}

Tests under adiabatic conditions are first presented in order to give preliminary information about the heat source and temperature variations in a simple case. Indeed, according to Eq. 31, we have $\dot{\theta}=s$. The temperature rate is equal to the heat source.

Figure 1 presents the evolution of the heat source $s$ and of the temperature variation $\theta$ over two mechanical cycles for test \#1 (uniaxial triangular signal). As expected for an entropic material, a certain amount of heat is produced during loading $[18 ; 19 ; 53]$. This quantity can be calculated by integrating the heat source over the loading duration. During unloading, the heat source is negative. The absolute value of the heat absorbed is equal to the heat produced during loading. As a consequence, the adiabatic nature of the evolution (no heat exchange with the outside) leads to a temperature variation equal to zero at the end of each mechanical cycle. As already mentioned in Section 3.2 (see Remark 2 above), the heat source value does not depend only on the stretch rate. It depends also on the value of stretch ratio $\lambda$ (see Eq. 24), so the heat source evolves during loading and unloading. For materials exhibiting only isentropic-type thermoelastic coupling (metallic materials in particular), the heat source is constant for triangular-shaped loadings, with only an opposite sign between loading and unloading. As the temperature variation oscillates here between 0 and a maximum value $\theta_{\max }$, the mean value between the maximum and minimum variations over a mechanical cycle is obviously different from zero: $\hat{\theta} \neq 0$.

Figure 2 shows the results obtained for test \#2 (uniaxial sinusoidal signal). The shape of the heat source curve is not exactly sinusoidal. This is clearly visible at low strains. A nearly horizontal plateau can be seen around zero deformation. An important remark can be made here. This type of response could be wrongly attributed to the effect of the variation in internal energy at low strains (isentropic coupling), but let us recall that isentropic coupling is not considered in the modeling here. Besides, isentropic coupling would have led to opposite signs for the heat sources (negative during loading and positive during unloading). See also Section 5.4. As a result, the temperature variation at low strain levels is affected by a particularity of the variation in heat sources: we can see a "flat valley" around zero deformation. This thermal response was observed experimentally in Ref. [25]. 


\subsection{Tests under non-adiabatic conditions}

In the present section, non-adiabatic conditions are considered to investigate the stabilized thermal regime.

Let us recall that the intrinsic dissipation is equal to zero in all our simulations $\left(\mathcal{D}_{\text {int }}=0\right)$. In such a situation, it is worth noting that, for purely isentropic materials (metals in particular), heat exchanges with the outside environment of the specimen lead to a value of $\hat{\theta}$ equal to zero in the steady-state regime; the temperature variation $\theta$ oscillates symmetrically around zero after the transient regime. Let us now analyze the stabilized temperature variation for hyperelastic materials.

\subsubsection{Effect of non-adiabaticity itself}

Figure 3 presents the evolution of the temperature variation for a triangular signal under non-adiabatic conditions (test \#3). Two main results can be drawn from this figure:

- Quantity $\hat{\theta}$, which corresponds to the mean value between the maximum and minimum variations over a mechanical cycle, decreases over the duration of the test. This can be simply explained as follows. During the first loading stage, the temperature increases because the heat source is positive. However, a certain quantity of heat is given to the outside because the material is warmer than the outside (this heat corresponds to the temporal integration of $\theta / \tau$ ). Thus, the temperature at the end of the first loading stage is lower than the value that would be obtained for adiabatic test conditions. During unloading, the heat source is negative, leading to a temperature decrease. This phenomenon is amplified by the fact that the material continues to give heat to the outside as long as it is warmer than the outside (as long as $\theta / \tau$ is positive). As a consequence, the temperature variation at the end of the first mechanical cycle is lower than zero. This phenomenon continues throughout the cycles.

- It can be observed that the mean value $\hat{\theta}$ between the maximum and minimum variations over a mechanical cycle reaches a stabilized value which is different from zero: see Figure 4 which shows two stabilized cycles (at the end of test \#3). Here, $\hat{\theta}$ is equal to $0.4043^{\circ} \mathrm{C}$. This effect is due to the particular variation in the heat source over time. This result can be observed whenever the chosen time constant $\tau$ is different from zero. This is a significant result, typical of rubber-like materials. It is worth noting that this phenomenon is not due to intrinsic dissipation $\left(\mathcal{D}_{\text {int }}\right.$ equal to zero in the simulations).

Remark 3 - Unlike $\hat{\theta}$, the mean temperature variation over one cycle is equal to zero in the steadystate regime. Indeed, according to Eq. 31, we have $\theta=\tau(s-\dot{\theta})$. First, the temporal integration of the heat source $s$ over a mechanical cycle is equal to zero, i.e. the heat produced is equal to that absorbed. Second, in the steady-state regime, the temporal integration of $\dot{\theta}$ is also equal to zero. As a consequence, the mean temperature variation over one cycle is also equal to zero in the steady-state regime.

\subsubsection{Effect of signal shape}

This section will highlight the fact that the value of $\hat{\theta}$ in the steady-state regime depends on the signal shape. Test \#4 uses the same parameters as test \#3, but the signal shape is now sinusoidal. Figures 5 and 6 show the evolution of the temperature variation $\theta$ over the test and for two stabilized cycles (at the end of the test), respectively. As observed previously for a triangular signal shape, the value of $\hat{\theta}$ is different from zero in the steady-state regime. The stabilized value of $\hat{\theta}$ obtained here $\left(0.3317^{\circ} \mathrm{C}\right)$ is different from that obtained for the triangular signal $\left(0.4043^{\circ} \mathrm{C}\right)$. As a general result, the stabilized value of $\hat{\theta}$ depends on the signal shape.

\subsubsection{Effect of loading frequency}

All the previous tests were performed at $0.5 \mathrm{~Hz}$. Test \#5 is devoted to the effect of the loading frequency on the stabilized value of $\hat{\theta}$. Test \#5 uses the same parameters as test \#4 apart from the frequency, 
which varies between 0.001 and $0.2 \mathrm{~Hz}$. For $f=0.001 \mathrm{~Hz}$, the period of the mechanical signal is equal to $1000 \mathrm{~s}$ : this value is much greater than $3 \tau$, so the temperature variation evolution is influenced by the heat exchanges with the outside environment during the cycle. On the contrary, for $f=0.2 \mathrm{~Hz}$, the period is equal to $5 \mathrm{~s}$ : this value is much lower than $3 \tau$, so the heat exchanges are very low during one cycle. The stabilized value of $\hat{\theta}$ versus the frequency is given in Figure 7 . Two curves are plotted in this figure. They correspond to two different values of $N k T^{r e f}: 1$ and $2 \mathrm{MPa}$. Several comments can be made:

- the ratio between the two curves is the same as the ratio between the elastic parameters;

- by decreasing the frequency, the stabilized value of $\hat{\theta}$ tends towards zero. This was expected, as the test tends to be isothermal;

- by increasing the frequency from zero, the stabilized value of $\hat{\theta}$ first increases and then reaches an asymptotic value. Note that for $N k T^{r e f}=2 \mathrm{MPa}$, this value is equal to that obtained previously for test \#4 $\left(0.3317^{\circ} \mathrm{C}\right.$ at $\left.0.5 \mathrm{~Hz}\right)$;

- the maximum value of $\hat{\theta}$ is reached for high loading frequencies (such that the signal period is inferior to about $3 \tau$ ), for which the heat exchanges have insufficient time to occur over one cycle.

These results are of importance for the prediction of fatigue life. Indeed, most approaches based on heat build-up measurements to evaluate quickly the fatigue life of hyperelastic materials use cyclic tests between 0.5 and $10 \mathrm{~Hz}$. Our results show that the corresponding value of $\hat{\theta}$ is maximum in this range of frequencies and can be wrongly associated with heat build-up due to fatigue.

\subsubsection{Effect of strain ratio $R_{\epsilon}$}

Test \#6 is similar to test \#4 (in particular, both have the same stretch ratio amplitude), but the strain ratio $R_{\epsilon}$ is equal to $\frac{1}{2}$ instead of 0 . Results are presented in Figures 8 and 9. For isentropic materials such as metals, the temperature amplitude $\theta_{\max }-\theta_{\min }$ would be the same in the stabilized regime (the loading frequency of $0.5 \mathrm{~Hz}$ is high enough to be nearly in adiabatic conditions over one mechanical cycle) and $\hat{\theta}$ would be equal to zero. Comparing test \#4 (Fig. 6) and test \#6 (Fig. 9) shows that the temperature amplitudes $\theta_{\max }-\theta_{\min }$ are different. Moreover, the (non-null) stabilized value of $\hat{\theta}$ depends on the considered zone of strain-stress relationship. For the present simulations, $\hat{\theta}$ equals $0.2819^{\circ} \mathrm{C}$ for $\lambda$ oscillating between 1 and 3 , and $0.3317^{\circ} \mathrm{C}$ for $\lambda$ oscillating between 2 and 4 .

\subsection{Remark on the effect of the multiaxiality of the mechanical state}

Tests \#4, \#7 and \#8 investigate the effect of three loading conditions, uniaxial tension (UT), equibiaxial tension (EQT) and pure shear (PS) respectively, classically used to characterize the mechanical response of rubber-like materials. Figure 10 groups the three stabilized thermal responses. As expected from the form of Eqs. 26 to 28, UT and PS exhibit very close heat source values, while the heat sources for EQT are approximately two times greater. For the present numerical simulations, the following results are found in the steady-state regime: $\hat{\theta}=0.3317^{\circ} \mathrm{C}$ for $\mathrm{UT}, 0.3287^{\circ} \mathrm{C}$ for PS and $1.2508^{\circ} \mathrm{C}$ for EQT.

\subsection{Remark on the effect of the internal energy variation}

As previously reported in Refs. [19] and [54], vulcanized rubber is characterized by slight cooling during stretching in the very low strain range (typically for $\lambda<1.1$ ), followed by strong heating when continuing the stretch increase. This phenomenon is referred to as the Gough-Joule effect in the literature and leads to a thermoelastic inversion. Indeed, a competition takes place between the effect of the internal energy variation (isentropic coupling) and the effect of entropy variation (entropic coupling). At low strain levels, the heat source is mainly composed of the contribution of the internal energy variation (negative heat source for a positive strain rate). At higher strain levels, the heat source is mainly composed of the contribution of the entropy variation (positive heat source for a positive strain rate). However, both contributions exist whatever the strain level. For instance, it can be seen 
from Eqs. 26-28 that the heat source due to entropic coupling is different from zero for small strains, too, i.e. as soon as the strain is different from zero. As a consequence, even for cyclic tests at small strains, the value of $\hat{\theta}$ is different from zero in the steady-state regime.

The contribution of the internal energy variation is rarely taken into account in the literature, although it significantly affects the thermal response of rubber at low strains. Ref. [24] proposed a modified entropic theory to account for this phenomenon using a volumetric part of the strain energy density that contains an energetic contribution to the heat source produced by the material. Figure 11 presents the evolution of the stretch ratio, the heat source and the temperature variation with test conditions similar to test \#4 but including the effect of the internal energy variation. The heat source variation is in correct agreement with the experimental results obtained at low strains by Pottier et al. [25]. The stabilized value of $\hat{\theta}$ is different from zero: it is here equal to $0.3261^{\circ} \mathrm{C}$. This value is slightly lower than that obtained for test \#4 $\left(0.3317^{\circ} \mathrm{C}\right)$. Isentropic coupling does not strongly change the conclusions.

\section{Conclusion}

This paper has investigated the thermal stabilization of rubber-like material under cyclic loading conditions. Results have shown that the mean value between the maximum and the minimum temperature variations differs from zero in the steady-state regime. This is due to the particular distribution of the heat source over time, and more especially to the fact that the heat source depends on both the strain and the strain rate. This phenomenon is observed while the temporal integration of the heat source is null over each cycle and no intrinsic dissipation occurs. This effect clearly distinguishes rubber from most other materials, whose thermoelastic response is driven by isentropic coupling. Moreover, the stabilized mean value between the maximum and minimum temperature variations depends on the strain ratio, the frequency and the multiaxiality of the mechanical state. The special thermosensitivity of rubber engenders many consequences, in particular for fatigue life prediction. Indeed, the stabilized mean value between the maximum and minimum temperature variations during fatigue tests does not reflect only self-heating due to fatigue, since entropic coupling leads also to a value that is not equal to zero. Finally, let us recall that small strain levels were investigated in the present study, leading to small thermal effects of the entropic coupling (but important for fatigue prediction). The effect would be much higher for large loading amplitudes, which besides could have an influence on thermo-oxidation of rubber. 


\section{References}

1. Mullins L (1948) Effect of stretching on the properties of rubber. Rubber Chemistry and Technology, 21:281300 .

2. Marckmann G, Verron E, Gornet L, Chagnon G, Charrier P, Fort P (2002) A theory of network alteration for the Mullins effect. Journal of the Mechanics and Physics of Solids, 50:2011-2028.

3. Diani J, Fayolle B, Gilormini P (2009) A review on the mullins effect. European Polymer Journal, 45:601612.

4. Fletcher WP, Gent AN (1953) Non-linearity in the dynamic properties of vulcanised rubber compounds. Trans Inst Rubber Ind, 29:266-280.

5. Payne AR (1962) The dynamic properties of carbon black-loaded natural rubber vulcanizates. part i. J Appl Phys, 6(19):57-63.

6. Rendek M, Lion A (2010) Strain-induced transient effects of filler-reinforced elastomers with respect to the Payne-effect: experiments and constitutive modelling. Z Angew Math Mech, 90:436-458.

7. Barick AK, Tripathy DK (2010) Thermal and dynamic mechanical characterization of thermoplastic polyurethane/organoclay nanocomposites prepared by melt compounding. Materials Science and Engineering: A, 527:812-823.

8. Barmouz M, Seyfi J, Kazem Besharati Givi M, Hejazi I, Davachi SM (2011) A novel approach for producing polymer nanocomposites by in-situ dispersion of clay particles via friction stir processing. Materials Science and Engineering: A, 528:3003-3006.

9. Stringfellow R, Abeyaratne R (1989) Cavitation in an elastomer: Comparison of theory with experiment. Materials Science and Engineering: A, 112:127-131.

10. Le Cam J-B, Toussaint E (2008) Volume variation in stretched natural rubber: competition between cavitation and stress-induced crystallization. Macromolecules, 41:7579-7583.

11. Le Cam J-B, Toussaint E (2009) Cyclic volume changes in rubbers. Mechanics of Materials, 41:898-901.

12. Toki S, Fujimaki T, Okuyama M (2000) Strain-induced crystallization of natural rubber as detected realtime by wide-angle x-ray diffraction technique. Polymer, 41:5423-5429.

13. Toki S, Sics I, Ran S, Liu L, Hsiao BS, Murakami S, Senoo K, Kohjiya S. Macromolecules, 35:6578-6584.

14. Trabelsi S, Albouy P-A, Rault J (2002) Stress-induced crystallization around a crack tip in natural rubber. Macromolecules, 35:10054-10061, 2002.

15. Trabelsi S, Albouy P-A, Rault J (2003) Effective local deformation in stretched filled rubber. Macromolecules, 36:9093-9099.

16. Huneau B (2011) Strain-induced crystallization of natural rubber: a review of x-ray diffraction investigations. Rubber Chemistry and Technology, 84:425-452.

17. Lion A, Peters J (2010) Coupling effects in dynamic calorimetry: frequency-dependent relations for specific heat and thermomechanical responses -a one-dimensional approach based on thermodynamics with internal state variables. Thermochim Acta, 500:76-87.

18. Gough J (1805) Proc Lit and Phil Soc Manchester, 2nd, ser. 1, page 288.

19. Joule JP (1857) On some thermodynamic properties of solids. Phil Mag 4th, 14:227.

20. Mars WV, Fatemi A (2002) A literature survey on fatigue analysis approaches for rubber. International Journal of Fatigue, 24:949-961.

21. Mars WV (2002) Cracking energy density as a predictor of fatigue life under multiaxial conditions. Rubber Chemistry and Technology, 75:1-17.

22. Le Cam J-B (2005) Endommagement en fatigue des elastomères. PhD thesis, Université de Nantes, École Centrale de Nantes.

23. Saintier N, Cailletaud G, Piques R (2006) Multiaxial fatigue life prediction for a natural rubber. International Journal of Fatigue, 28:530-539.

24. Chadwick P, Creasy CMF (1984). Modified entropic elasticity of rubberlike materials. Journal of Mechanics and Physics of Solids, 32:337-357.

25. Pottier T, Moutrille M-P, Le Cam J-B, Balandraud X, Grédiac M (2009) Study on the use of motion compensation technique to determine heat sources. application to large deformations on cracked rubber specimens. Experimental Mechanics, 49:561-574.

26. Promma N, Raka B, Grédiac M, Toussaint E, Le Cam J-B, Balandraud X, Hild F (2009) Application of the virtual fields method to mechanical characterization of elastomeric materials. International Journal of Solids and Structures, 46:698-715.

27. Le Cam J-B, Toussaint E, Dubois O (2012) Effect of thermal cycles on the deformation state at the crack tip of crystallizable natural rubber. Strain, 48:153-156.

28. Le Cam J-B (2012) A review of the challenges and limitations of full-field measurements applied to large heterogeneous deformation of rubbers. Strain, 48:174-188.

29. Toussaint E, Balandraud X, Le Cam J-B, Grédiac M (2012) Combining displacement, strain, temperature and heat source fields to investigate the thermomechanical response of an elastomeric specimen subjected to large deformations. Polymer Testing, 31:916-925.

30. Verron, E, Le Cam J-B, Gornet, L (2006) A multiaxial criterion for crack nucleation in rubber Mechanics Research Communications, 33:493-498.

31. Samaca Martinez JR, Le Cam J-B, Balandraud X, Toussaint E, Caillard J (2013) Mechanisms of deformation in crystallizable natural rubber. Part 2: quantitative calorimetric analysis. Polymer, 54:2727-2736.

32. Samaca Martinez JR, Le Cam J-B, Balandraud X, Toussaint E, Caillard J (2013) Filler effects on the thermomechanical response of stretched rubbers. Polymer Testing, 32:835-841.

33. Lemaitre J, Chaboche JL (1990) Mechanics of Solids Materials. Cambridge University Press, Cambridge. 
34. Maugin GA (1999) The Thermodynamics of Nonlinear Irreversible Behaviors: an Introduction, volume 27 of Series A. World scientific series on Nonlinear Science.

35. Holzapfel GA(2000) Non linear solid mechanics - a continuum approach for engineering.

36. Chrysochoos A (1995) Analyse du comportement des matériaux par thermographie infrarouge. Photomécanique 95, Y. Berthaud (ed.), Eyrolles, pp 203-211, Cachan, France, 14-16 March.

37. Chrysochoos A, Pham H, Maisonneuve O (1996) Energy balance of thermoelastic martensite transformation under stress. Nuclear Engineering Design, 162:1-12.

38. Balandraud X, Ernst E, Soos E (1999) Rheological phenomena in shape memory alloys. C. R. Acad. Sci., Ser. IIb Mec., 327:33-39.

39. Boulanger T, Chrysochoos A, Mabru C, Galtier A (2004) Calorimetric analysis of dissipative and thermoelastic effects associated with the fatigue behavior of steels. International Journal of Fatigue, 26:221-229.

40. Berthel B, Chrysochoos A, Wattrisse B, Galtier A (2008) Infrared image processing for the calorimetric analysis of fatigue phenomena. Experimental Mechanics, 48:79-90.

41. Giancane S, Chrysochoos A, Dattoma V, Wattrisse B (2009) Deformation and dissipated energies for high cycle fatigue of 2024-T3 aluminium alloy. Theoretical and Applied Fracture Mechanics, 52:117-121.

42. Chrysochoos A, Louche H (2000) An infrared image processing to analyse the calorific effects accompanying strain localisation. Int J Eng Sci, 38:1759-1788.

43. Chrysochoos A, Louche H (2001) Thermal and dissipative effects accompanying luders band propagation. Mat Sci Eng A-struct, 307:15-22.

44. Balandraud X, Chrysochoos A, Leclercq S, Peyroux R (2001) Influence of the thermomechanical coupling on the propagation of a phase change front. C R Acad Sci - Series IIB - Mechanics, 329:621-626.

45. Chrysochoos A, Wattrisse B, Muracciole J-M, El Kaim Y (2009) Fields of stored energy associated with localized necking of steel. J. of Mech. of Mater. and Struct., 4:245-262.

46. Maquin F, Pierron F (2009) Heat dissipation measurements in low stress cyclic loading of metallic materials: From internal friction to micro-plasticity. Mechanics of Materials, 41:928-942.

47. Dumoulin S, Louche H, Hopperstad OS, Borvik T (2010) Heat sources, energy storage and dissipation in high-strength steels: Experiments and modelling. European Journal of Mechanics A-Solids, 29:461-474.

48. Verron E, Andriyana A (2008) Definition of a new predictor for multiaxial fatigue crack nucleation in rubber. Journal of the Mechanics and Physics of Solids, 56:417-443.

49. Baaser H, Hopmann C, Schobel A (2013) Reformulation of strain invariants at incompressibility. Archive of Applied Mechanics, 83:273-280.

50. Meunier L, Chagnon G, Favier D, Orgéas L, Vacher P (2008) Experimental and numerical study of the mechanical behaviour of an unfilled silicone rubber. Polymer Testing, 27:765-777.

51. Treloar LRG (1943) The elasticity of a network of long chain molecules (I and II). Transactions of the Faraday Society, 39:36-64;241-246.

52. Dulieu-Barton JM, Stanley P (1998) Development and application of thermoelastic stress analysis. Journal of Strain Analysis and Engineering Design, 33:93-104.

53. Joule JP (1884) India-rubber, $§ 33$ in: The Scientific Papers of James Prescott Joule, volume 1. The Physical Society of London, Taylor and Francis, red lion court, fleet street.

54. Anthony RL, Caston RH, Guth E (1942) Equations of state for naturals and synthetic rubber like materials: unaccelerated natural soft rubber. Journal of Physical Chemistry, 46:826. 

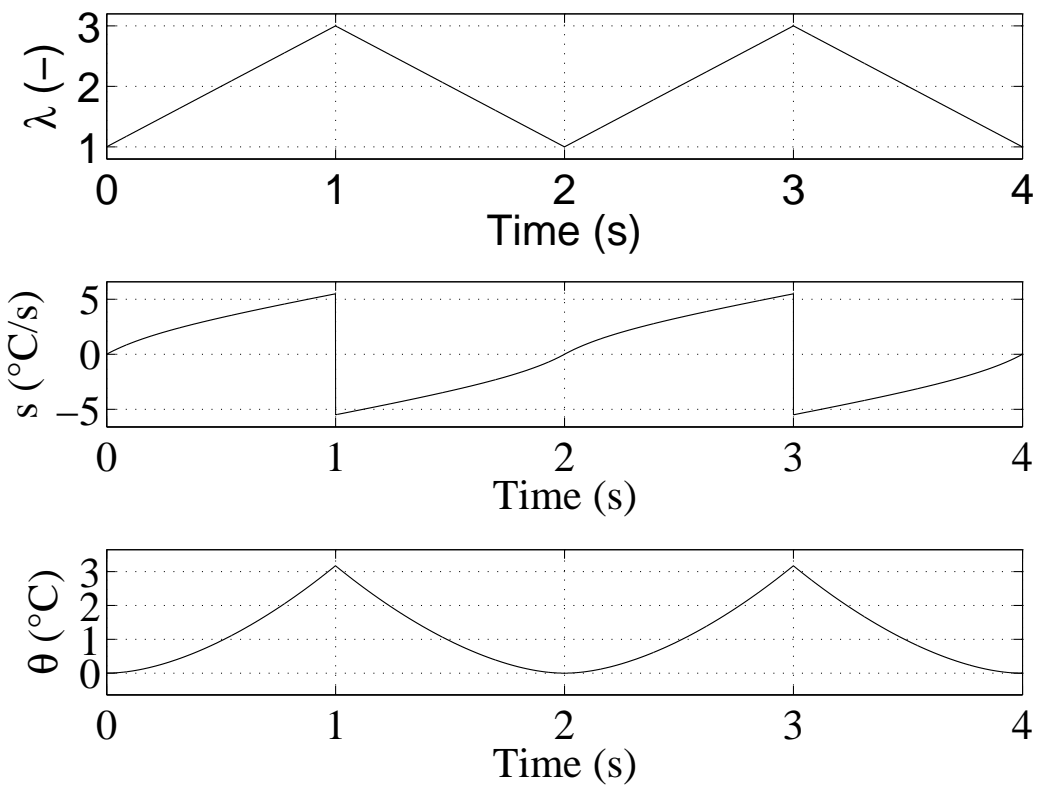

Fig. 1 Test \#1 (adiabatic conditions, triangular signal, $\lambda_{\min }=1, \lambda_{\max }=3, R_{\epsilon}=0, f=0.5 \mathrm{~Hz}$ ). Top: stretch ratio $\lambda$ vs. time. Middle: heat source $s$ vs. time. Bottom: temperature variation $\theta$ vs. time.
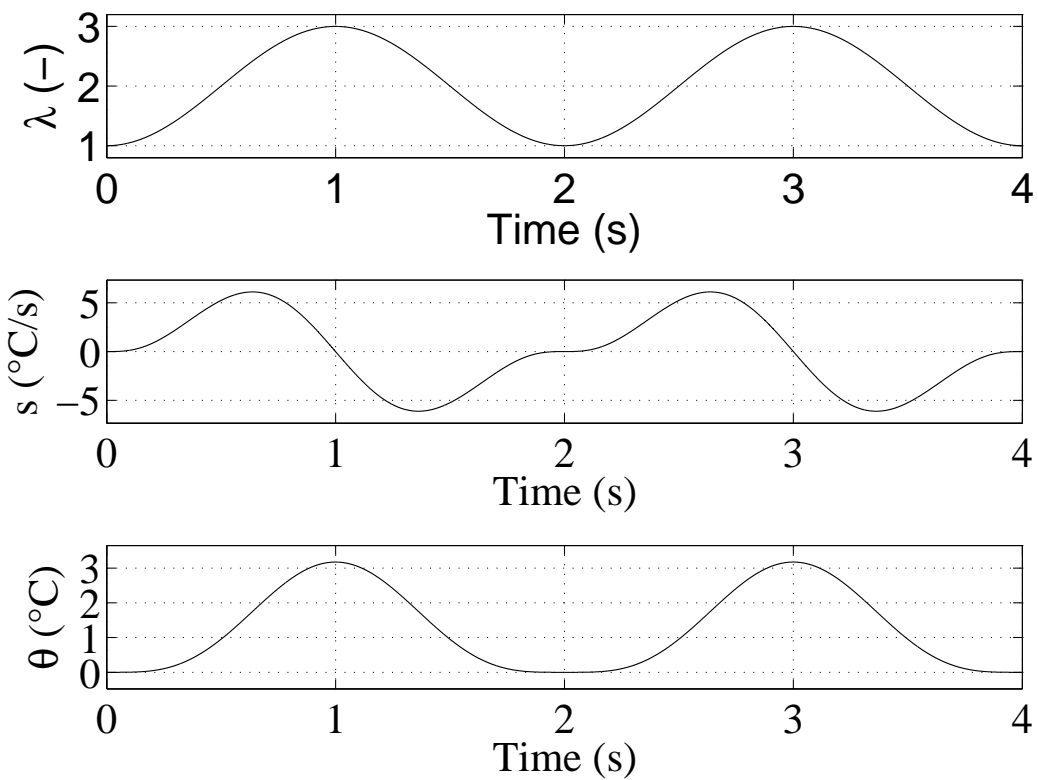

Fig. 2 Test \#2 (adiabatic conditions, sinusoidal signal, $\lambda_{\min }=1, \lambda_{\max }=3, R_{\epsilon}=0, f=0.5 H z$ ). Top: stretch ratio $\lambda$ vs. time. Middle: heat source $s$ vs. time. Bottom: temperature variation $\theta$ vs. time. 


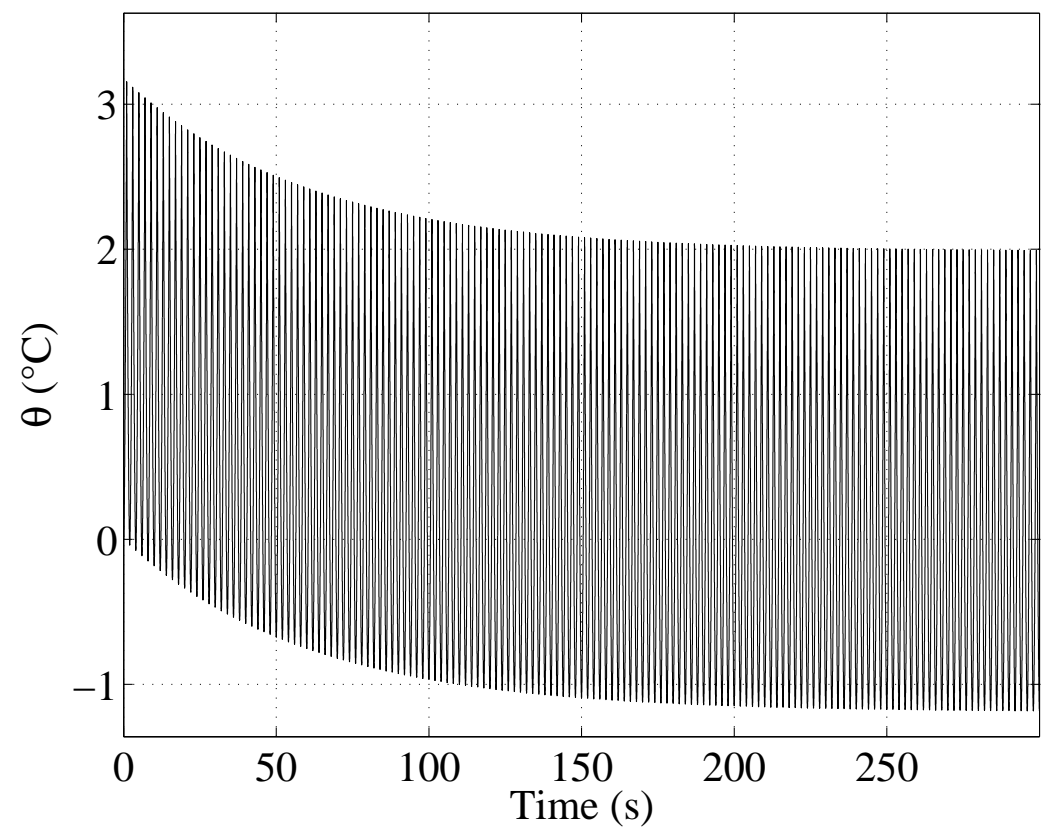

Fig. 3 Test \#3 (non-adiabatic conditions, triangular signal, $\lambda_{\min }=1, \lambda_{\max }=3, R_{\epsilon}=0, f=0.5 H z$ ). Temperature variation $\theta$ vs. time.
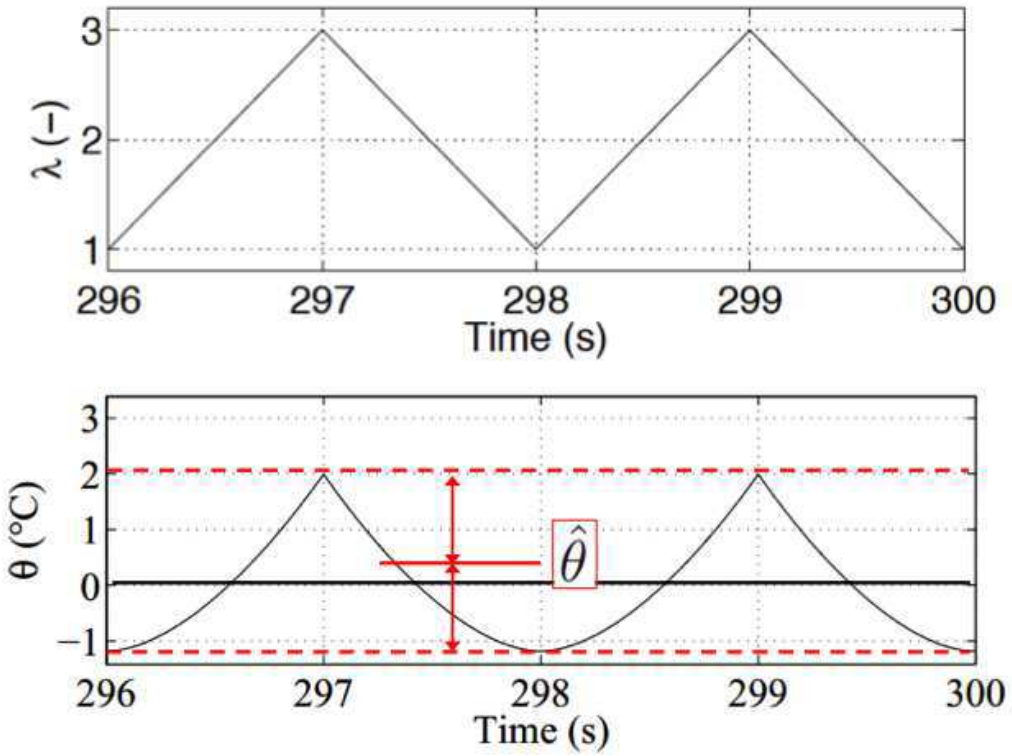

Fig. 4 Last two cycles of test \#3. 


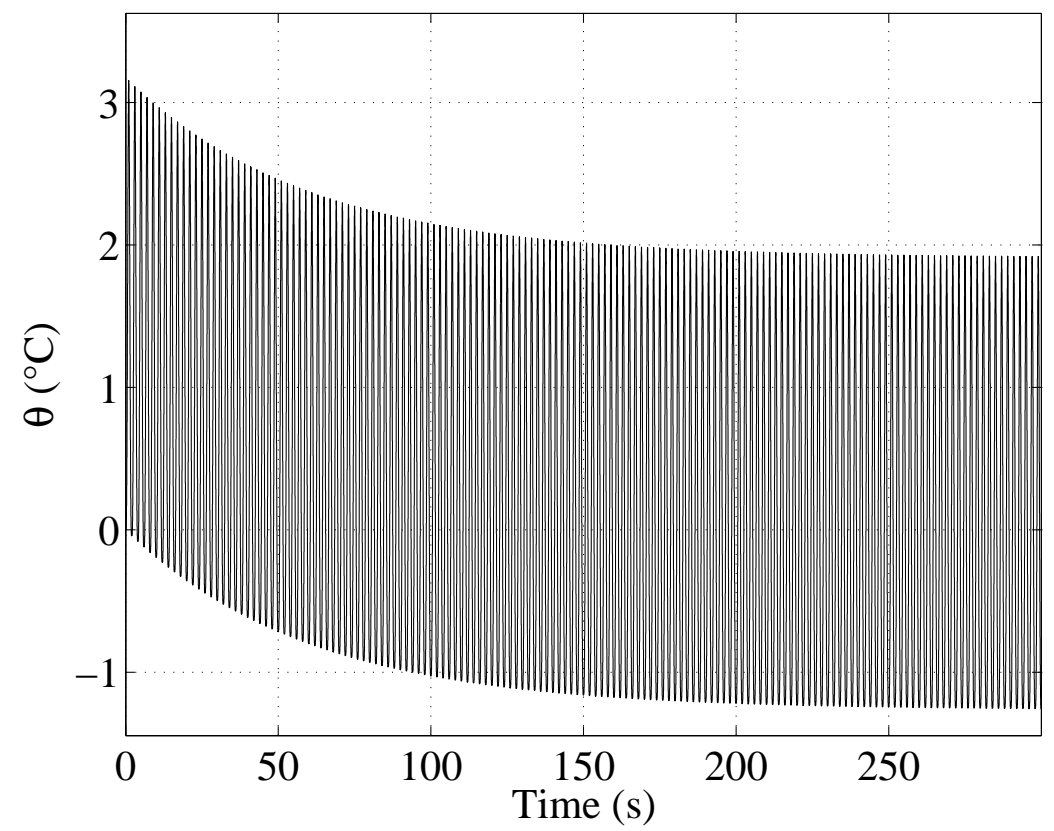

Fig. 5 Test \#4 (non-adiabatic conditions, sinusoidal signal, $\lambda_{\min }=1, \lambda_{\max }=3, R_{\epsilon}=0, f=0.5 H z$ ). Temperature variation $\theta$ vs. time.


Fig. 6 Last two cycles of test \#4. 


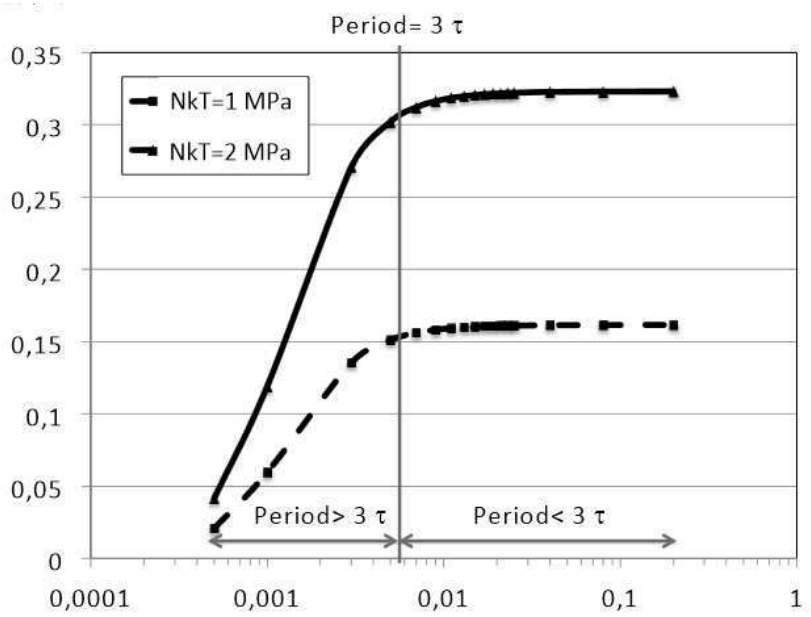

Fig. 7 Test \#5: stabilized value of $\hat{\theta}$ versus loading frequency.

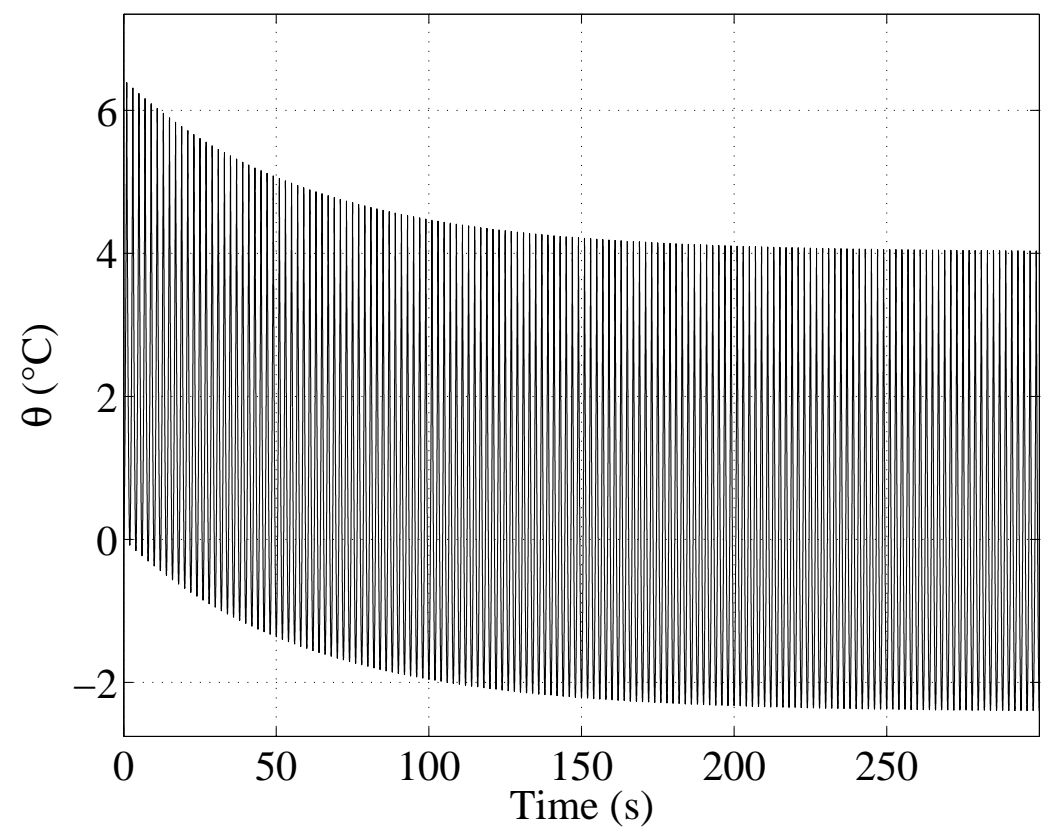

Fig. 8 Test \#6 (non-adiabatic conditions, sinusoidal signal, $\lambda_{\min }=2, \lambda_{\max }=4, R_{\epsilon}=1 / 2, f=0.5 H z$ ). Top: stretch ratio $\lambda$ vs. time. Middle: heat source $s$ vs. time. Bottom: temperature variation $\theta$ vs. time. 

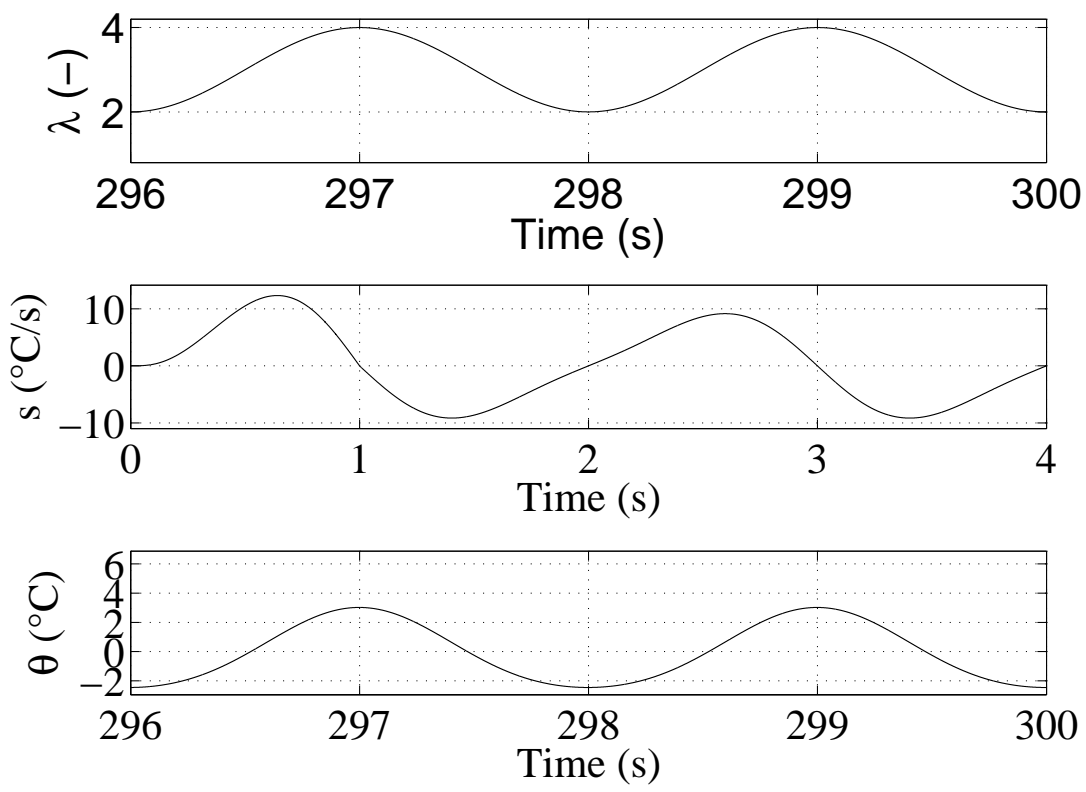

Fig. 9 Last two cycles of test \#6.
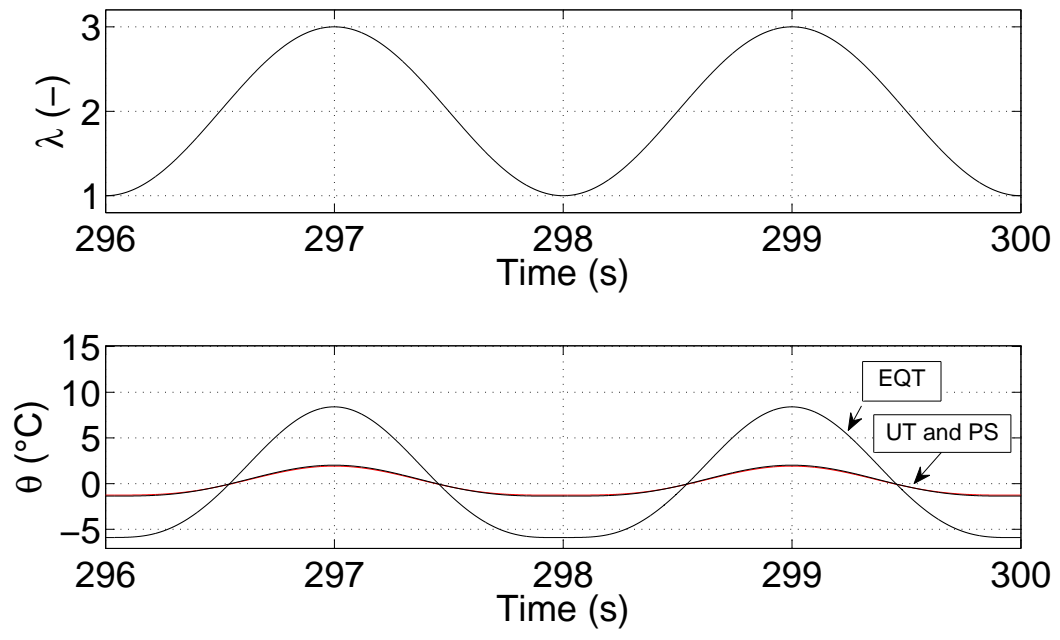

Fig. 10 Results obtained under uniaxial tension (UT), pure shear (PS) and equib- iaxial tension (EQT) for the last two cycles for non-adiabatic conditions. The other parameters are those used for test \#4. Top: stretch ratio $\lambda$ vs. time. Bottom: temperature variation $\theta$ vs. time.

Table 1 Uniaxial tensile (UT) tests under adiabatic conditions $(\tau=\infty)$

\begin{tabular}{llllll}
\hline Test number & $\lambda_{\min }[-]$ & $\lambda_{\max }[-]$ & $R_{\epsilon}[-]$ & $f(\mathrm{~Hz})$ & Signal shape \\
\hline$\# 1$ & 1 & 3 & 0 & 0.5 & triangular \\
$\# 2$ & 1 & 3 & 0 & 0.5 & sinusoidal \\
\hline
\end{tabular}



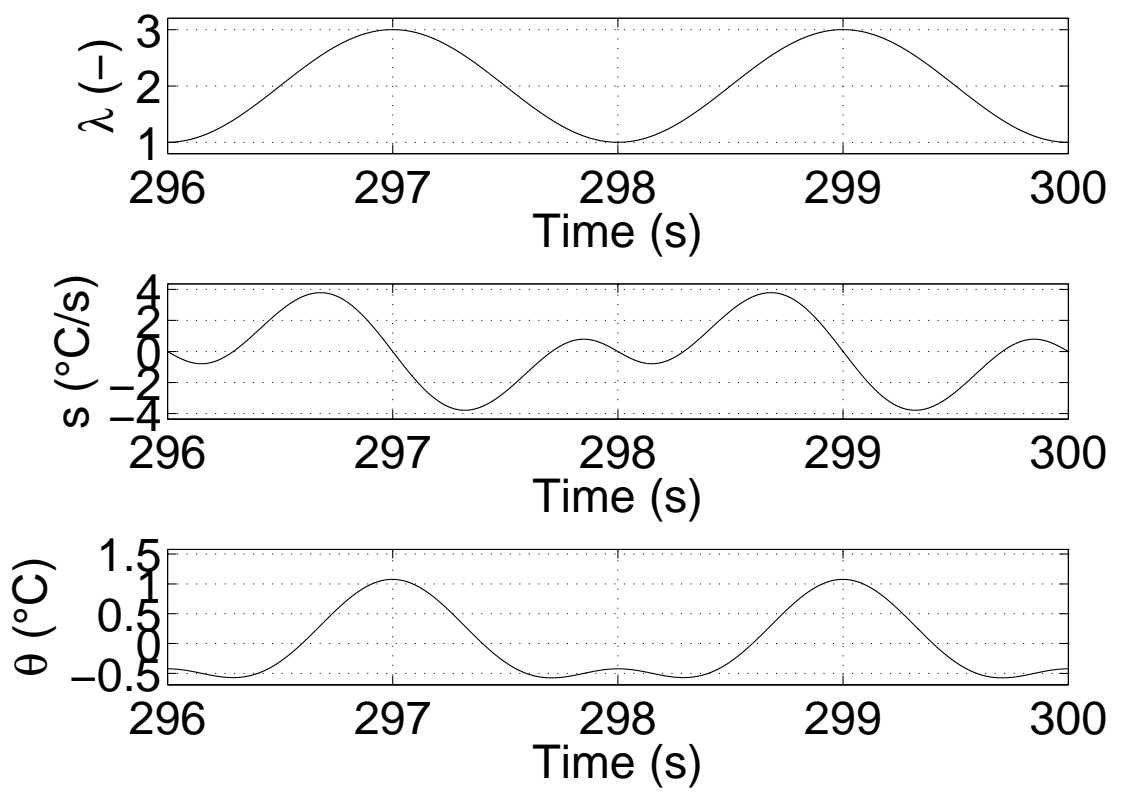

Fig. 11 Test similar to test \#2 but accounting for isentropic coupling. Top: stretch ratio $\lambda$ vs. time. Middle: heat source $s$ vs. time. Bottom: temperature variation $\theta$ vs. time.

Table 2 Mechanical tests under non-adiabatic conditions $(\tau=60 \mathrm{~s})$

\begin{tabular}{lllllll}
\hline $\begin{array}{l}\text { Test } \\
\text { number }\end{array}$ & $\begin{array}{l}\lambda_{\min } \\
{[-]}\end{array}$ & $\begin{array}{l}\lambda_{\max } \\
{[-]}\end{array}$ & $\begin{array}{l}R_{\epsilon} \\
{[-]}\end{array}$ & $\begin{array}{l}f \\
(\mathrm{~Hz})\end{array}$ & $\begin{array}{l}\text { Signal } \\
\text { shape }\end{array}$ & $\begin{array}{l}\text { Loading } \\
\text { conditions }\end{array}$ \\
\hline$\# 3$ & 1 & 3 & 0 & 0.5 & triangular & $\mathrm{UT}$ \\
$\# 4$ & 1 & 3 & 0 & 0.5 & sinusoidal & $\mathrm{UT}$ \\
$\# 5$ & 1 & 3 & 0 & {$[0.001 ; 0.2]$} & sinusoidal & $\mathrm{UT}$ \\
$\# 6$ & 2 & 4 & $\frac{1}{2}$ & 0.5 & sinusoidal & UT \\
$\# 7$ & 1 & 3 & 0 & 0.5 & sinusoidal & EQT \\
$\# 8$ & 1 & 3 & 0 & 0.5 & sinusoidal & PS \\
\hline
\end{tabular}

